Article

\title{
Electronic and Magnetic Properties of Ni-Doped Zinc-Blende ZnO: A First-Principles Study
}

\author{
Suqin Xue ${ }^{1}$, Fuchun Zhang ${ }^{2, *}$, Shuili Zhang ${ }^{2}$, Xiaoyang Wang ${ }^{2}$ and Tingting Shao ${ }^{2}$ \\ 1 College of Mathematics and Computer Science, Yan'an University, Yan'an 716000, Shanxi, China; \\ ydxsq@yau.edu.cn \\ 2 College of Physics and Electronic Information, Yan'an University, Yan'an 716000, Shanxi, China; \\ zhangshuili74@163.com (S.Z.); yettawang15@163.com (X.W.); retastt@126.com (T.S.) \\ * Correspondence: yadxzfc@yau.edu.cn; Tel.: +86-911-233-2045
}

Received: 11 March 2018; Accepted: 24 April 2018; Published: 26 April 2018

\begin{abstract}
The electronic structure, band structure, density of state, and magnetic properties of Ni-doped zinc-blende (ZB) $\mathrm{ZnO}$ are studied by using the first-principles method based on the spin-polarized density-functional theory. The calculated results show that $\mathrm{Ni}$ atoms can induce a stable ferromagnetic (FM) ground state in Ni-doped ZB ZnO. The magnetic moments mainly originate from the unpaired $\mathrm{Ni} 3 d$ orbitals, and the $\mathrm{O} 2 p$ orbitals contribute a little to the magnetic moments. The magnetic moment of a supercell including a single Ni atom is $0.79 \mu_{\mathrm{B}}$. The electronic structure shows that Ni-doped $\mathrm{ZB} \mathrm{ZnO}$ is a half-metallic FM material. The strong spin-orbit coupling appears near the Fermi level and shows obvious asymmetry for spin-up and spin-down density of state, which indicates a significant hybrid effects from the Ni $3 d$ and $\mathrm{O} 2 p$ states. However, the coupling of the anti-ferromagnetic (AFM) state show metallic characteristic, the spin-up and spin-down energy levels pass through the Fermi surface. The magnetic moment of a single $\mathrm{Ni}$ atom is $0.74 \mu_{\mathrm{B}}$. Moreover, the results show that the Ni $3 d$ and $\mathrm{O} 2 p$ states have a strong $p-d$ hybridization effect near the Fermi level and obtain a high stability. The above theoretical results demonstrate that $\mathrm{Ni}$-doped zinc blende $\mathrm{ZnO}$ can be considered as a potential half-metal FM material and dilute magnetic semiconductors.
\end{abstract}

Keywords: Ni-doped; $\mathrm{ZnO}$; first-principles; ferromagnetic and anti-ferromagnetic

\section{Introduction}

Diluted magnetic semiconductors (DMSs) are a new type of semiconductor material which obtain FM properties by doping $3 d$ transition-metal atoms. They have been investigated extensively because of their potential usage of both the charge and spin properties of freedom of carriers in spintronic devices. Many researchers expected to be able to prepare ultra-high speed, super bandwidth, low-power, and non-volatile spintronic devices based on DMSs, such as spin field effect transistors (Spin-FET), spin light-emitting diodes (Spin-LED), spin resonant tunneling diodes (Spin-RTD), optical isolators, magnetic sensors, nonvolatile memory cells, etc., [1-8]. During the past years, the investigation into the applications of $\mathrm{ZnO}$ nano-semiconductor materials has become a hot topic in the wide-gap semiconductor materials field $[9,10]$. $\mathrm{ZnO}$ materials have n-type semiconductor characteristics due to the inherent oxygen vacancy and other intrinsic n-type defects, because of this, $\mathrm{ZnO}$ materials are potential DMSs materials with a high Curie temperature. Sato et al. [11] have researched transition-metal atoms $(\mathrm{Mn}, \mathrm{V}, \mathrm{Cr}, \mathrm{Fe}, \mathrm{Co}, \mathrm{Ni})$ doped $\mathrm{ZnO}$ materials through first-principle calculations, and the results show that the doping system has FM properties. Ueda et al. [12] have also successfully prepared $3 d$ transition-metal-doped $\mathrm{ZnO}$ thin film materials, the results show that the Curie temperature is greater than $280 \mathrm{~K}$. Herein, we especially highlight Igamberdiev's 
work [13], providing and reporting the magnetic phase transitions in ZnO doped with Mn for DMSs. Liu et al. [14] have predicted the possibility of ferromagnetism in wide-band gap semiconductors $\mathrm{ZnO}$ and $\mathrm{GaN}$ based on the recent progress in the theoretical and experimental studies of $\mathrm{ZnO}-$ and GaN-based DMSs. In terms of Ni-doped $\mathrm{ZnO}$ research, Zhao has reported room temperature FM in $\mathrm{Zn}_{1-x} \mathrm{Ni}_{x} \mathrm{O}(x=0.1 \%, 0.4 \%, 0.7 \%, 1.0 \%)$ powders using the sol-gel technique [15]. Liu et al. [16] have successfully synthesized Ni-doped ZnO with pulsed laser deposition (PLD), the results show that $\mathrm{Ni}$-doped $\mathrm{ZnO}$ has FM properties. Cui et al. [17] have synthesized Ni- and Co-doped ZnO nanowire arrays with an electrochemical method at $90{ }^{\circ} \mathrm{C}$, and the results show that magnetic nanowires have anisotropic FM properties. Wang et al. [18] have prepared Ni-doped ZnO nanowire arrays by means of metal vapor vacuum arc (MEVVA) ion source doping technology, and the results show that the electron transport ability increases by 30 times and the absorption peak exhibits red-shift phenomena. Wakano et al. [19] have also synthesized Ni-doped ZnO thin film materials, and the results show that FM features appear at $2 \mathrm{~K}$, while PM properties appear at $300 \mathrm{~K}$. Al-Harbihas synthesized a $\mathrm{Zn}_{1-x} \mathrm{Ni}_{x} \mathrm{O}$ nanorod with excellent UV emissive power by means of a low-temperature hydrothermal method [20]. Cheng et al. have also prepared a $\mathrm{Zn}_{1-x} \mathrm{Ni}_{x} \mathrm{O}$ nanorod with room temperature FM features by means of a low-temperature hydrothermal method [21]. However, Yin et al. have reported that no FM feature has been observed in $\mathrm{Zn}_{1-x} \mathrm{Ni}_{x} \mathrm{O}$ nano-materials [22].

Until now, most studies have been focused on $3 d$ transition-metal-doped wurtzite $\mathrm{ZnO}$ materials, including synthesis, theoretical prediction, and the FM coupling mechanism of DMSs at room temperature. However, theoretical or experimental study on transition-metal atom-doped $\mathrm{ZB} \mathrm{ZnO} \mathrm{DMSs} \mathrm{is} \mathrm{uncommon.} \mathrm{Most} \mathrm{research} \mathrm{studies} \mathrm{show} \mathrm{that} \mathrm{the} \mathrm{samples} \mathrm{prepared} \mathrm{with}$ transition-metal-doped $\mathrm{ZnO}$ materials vary greatly in performance, being poor in experiment repeatability. Meanwhile, the magnetic origin and magnetic coupling mechanism should also be further studied.

In this paper, we replace the two nearest neighboring (Ni-O-Ni) and the next nearest neighboring (Ni-O-Zi-O-Ni) Zn atoms in different positions with two Ni atoms. The purpose of our work is to study the electronic structures, band structure, density of state, FM and AFM properties, and magnetic coupling mode of $\mathrm{Ni}$-doped $\mathrm{ZB} \mathrm{ZnO}$ using the density functional theory, and reveal the magnetic origin and FM coupling mechanism. The research results will provide a theoretical basis for the application of $\mathrm{ZnO}$ DMSs.

\section{Theoretical Models and Calculated Methods}

To study the electronic structure and the magnetism of Ni-doped ZB ZnO, we constructed the model of Ni-doped ZB ZnO [23], as shown in Figure 1a. The doping configurations of Ni-doped ZB $\mathrm{ZnO}$ are based on the $2 \times 2 \times 2 \mathrm{ZB}$ supercell containing $32 \mathrm{Zn}$ atoms and $32 \mathrm{O}$ atoms with a doping concentration of 6.25 at. \%. In order to study the stability of coupling magnetic interactions in the ground state, two different spatial constructs (refered to as configuration I $(1,2)$ and II $(1,3)$ ) based on the Ni substitution sites were studied, where the two nearest-neighboring or next nearest-neighboring $\mathrm{Zn}$ atoms were replaced by two $\mathrm{Ni}$ atoms. In configuration $\mathrm{I}$, the two neighboring Ni atoms were direct coupling through a single $\mathrm{O}(\mathrm{Ni}-\mathrm{O}-\mathrm{Ni})$ atom at the minimum distance. In configuration II, The two neighboring $\mathrm{Ni}$ atoms were indirect coupling through an atomic chain including two $\mathrm{O}$ atoms and one $\mathrm{Zn}$ atoms (Ni-O-Zn-O-Ni) at a comparatively larger distance. In addition, in order to confirm the suitability of the employed supercells, we also constructed a $1 \times 2 \times 2$ supercell containing $16 \mathrm{Zn}$ atoms and $16 \mathrm{O}$ atoms (Figure $1 \mathrm{~b}$ ); the model adopted the same doping method as the $2 \times 2 \times 2 \mathrm{ZB}$ supercell. These two different spatial constructs (refered to as configuration III $(1,2)$ and IV $(1,3))$ had a corresponding doping concentration of 12.5 at. \%. 


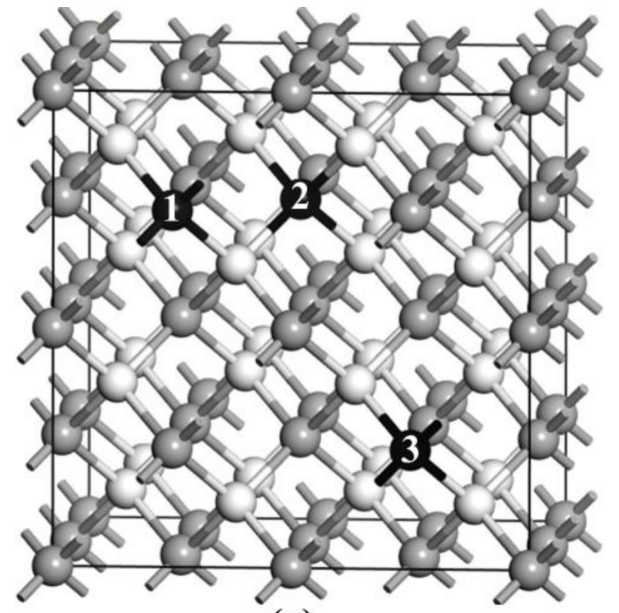

(a)

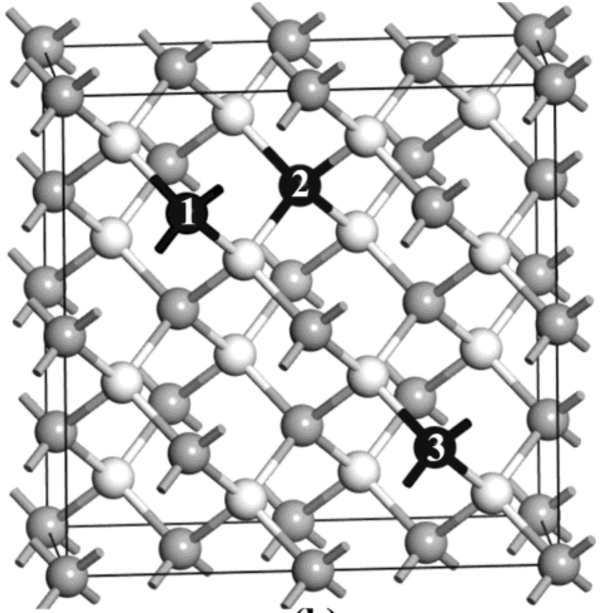

(b)

Figure 1. The crystal model of Ni-doped zinc-blende (ZB) $\mathrm{ZnO}$ (the gray and white balls indicate $\mathrm{Zn}$ and $\mathrm{O}$ atoms, respectively). The positions of $\mathrm{Zn}$ substituted by $\mathrm{Ni}$ are denoted by 1 and 2-3, (a) $2 \times 2 \times 2$ supercell; (b) $1 \times 2 \times 2$ supercell.

First-principles calculations based on the density functional theory (DFT) [24] with a plane-wave pseudo-potential basis were performed using the VASP software package [25] to study Ni-doped ZB $\mathrm{ZnO}$. The detailed parameters settings are shown as follows: the pseudo potential is treated by using projector augmented waves (PAW) potentials [26] to represent the interaction between electron and ionic. The electron exchange-correlation potential is performed by using the Perdew-Burke-Ernzerhof (PBE) formulation of Generalized Gradient Approximation (GGA). The valence electron configurations of $\mathrm{O}, \mathrm{Zn}$ and $\mathrm{Ni}$ atoms were performed as $2 s^{2} 2 p^{4}, 3 d^{10} 4 s^{2}$ and $3 d^{8} 4 s^{2}$, respectively. The cutoff energy of the plane-wave basis set was set at $420 \mathrm{eV}$. The self-convergence accuracy of the iterative process was set as less than $1 \times 10^{-6} \mathrm{eV} /$ atom. The maximal displacement convergence was $1 \times 10^{-4} \mathrm{~nm}$ and the internal stress was less than $0.1 \mathrm{GPa}$. For the Brillouin-zone integration, the $6 \times 6 \times 6 k$-point grids for the 64 atoms were employed by using a special $k$-points sampling scheme of Monkhorst-Pack in the reciprocal space.

\section{Results and Discussion}

Table 1 shows the lattice parameters of $\mathrm{Ni}$-doped $\mathrm{ZB}$ of the $2 \times 2 \times 2$ and $1 \times 2 \times 2$ supercells. For configuration I and configuration II, the lattice parameters $a, b$, and $c$ are significantly higher than that of pure $\mathrm{ZnO}$, indicating that the volume of $\mathrm{Ni}$-doped $\mathrm{ZB} \mathrm{ZnO}$ expands slightly. This phenomenon cannot be explained by conventional theories, because the $\mathrm{Ni}^{2+}$ ion radius $(0.69 \AA)$ is smaller than the $\mathrm{Zn}^{2+}$ ion radius $(0.74 \AA)$. We believe that there is no new compound formed after doping $\mathrm{Ni}^{2+}$ ion, but the replacement of $\mathrm{Zn}^{2+}$ with $\mathrm{Ni}^{2+}$ causes lattice distortion. As the $\mathrm{Ni}^{2+}$ ion radius is smaller than the $\mathrm{Zn}^{2+}$ ion radius, the residual stress generated during the crystallization process results in an increased mutual repulsion between the $\mathrm{Ni}^{2+}$ ion polarization charges, the system energy rises and so triggers the volume increase. The observed expansion in volume $V_{0}$ has also been found in nanoplatelets of $\mathrm{Ni}$-doped $\mathrm{ZnO}$ [27] and $\mathrm{Ni}$ - and Co-doped $\mathrm{ZnO}$ [28]. Moreover, for configuration III and configuration IV, the calculated lattice constants showed a good consistency with the $2 \times 2 \times 2$ supercell, and four configurations of $\mathrm{Ni}$-doped $\mathrm{ZB} \mathrm{ZnO}$ were approximate to the experimental value [23], the calculated relative error was less than $2 \%$. In order to satisfy the limit of doping concentration in experimental results, we adopted configuration I and configuration II to investigate the electronic and magnetic properties with a doping concentration of 6.25 at. $\%$. 
Table 1. Lattice constant of Ni-doped zinc-blende (ZB) ZnO.

\begin{tabular}{lcccc}
\hline Lattice Constant & Ni Position & $\mathbf{a}(\AA)$ & $\mathbf{b}(\AA)$ & $\mathbf{c}(\AA)$ \\
\hline $\mathrm{Zn}_{32} \mathrm{O}_{32}$ & - & 9.26 & 9.26 & 9.26 \\
Configuration I $\left(\mathrm{Zn}_{30} \mathrm{Ni}_{2} \mathrm{O}_{32}\right)$ & 1,2 & 9.5304 & 9.5493 & 9.5304 \\
Configuration II $\left(\mathrm{Zn}_{30} \mathrm{Ni}_{2} \mathrm{O}_{32}\right)$ & 1,3 & 9.5303 & 9.5303 & 9.5494 \\
Configuration III $\left(\mathrm{Zn}_{14} \mathrm{Ni}_{2} \mathrm{O}_{16}\right)$ & 1,2 & 4.6981 & 9.4918 & 9.4863 \\
Configuration IV $\left(\mathrm{Zn}_{14} \mathrm{Ni}_{2} \mathrm{O}_{16}\right)$ & 1,3 & 4.6893 & 9.4919 & 9.4939 \\
\hline
\end{tabular}

Table 2 shows the FM, AFM coupled configuration, bond length, and magnetic moments of $\mathrm{Ni}$-doped $\mathrm{ZB} \mathrm{ZnO;} \mathrm{Zn}-\mathrm{O}$ and $\mathrm{Ni}-\mathrm{O}$, respectively, are the neighbor bond lengths of $\mathrm{Zn}$. In order to determine the ground state of $\mathrm{Ni}$-doped $\mathrm{ZB} \mathrm{ZnO}$, the total energy and energy differences of the FM and AFM phases in the above configurations were calculated. The $\Delta E$ is the energy difference $\left(\Delta E=E_{A F M}-E_{F M}\right)$ of the AFM and FM states in relaxation. If the $\Delta E$ is smaller than zero, it indicates that the AFM state is more stable, if the $\Delta E$ is larger than zero, it indicates that the FM state is more stable. The total energies for configuration I and II are calculated as $-68055.9 \mathrm{eV}$ and $-68055.5 \mathrm{eV}$, respectively. The comparatively lower energy in configuration I indicated that Ni-doped ZB ZnO have a tendency to cluster together and favor short-range $\mathrm{Ni}-\mathrm{Ni}$ magnetic coupling, so the doping system is more likely to form impurity phases like $\mathrm{NiO}_{x}$. The observed phenomenon has also been found in other Ni-doped systems [29,30].

Table 2. Energy, magnetic coupling, bond length, and magnetic moments of Ni-doped ZB ZnO.

\begin{tabular}{cccccccccc}
\hline Composition & Coupling & $\begin{array}{c}\text { Energy } \\
(\mathbf{e V})\end{array}$ & $\begin{array}{c}\mathbf{N i}-\mathbf{O} \\
(\AA)\end{array}$ & $\begin{array}{c}\mathrm{Zn}-\mathbf{O} \\
(\AA)\end{array}$ & $\begin{array}{c}\mathbf{N i 1} \\
\left(\mu_{\mathrm{B}}\right)\end{array}$ & $\begin{array}{c}\mathbf{N i 2} \\
\left(\mu_{\mathrm{B}}\right)\end{array}$ & $\begin{array}{c}\mathbf{O} \\
\left(\mu_{\mathrm{B}}\right)\end{array}$ & Population & $\boldsymbol{\Delta} \boldsymbol{E}(\mathbf{e V})$ \\
\hline $\mathrm{Zn}_{32} \mathrm{O}_{32}$ & - & -68072.1 & - & 2.003 & - & - & - & 0.35 & - \\
Configuration I & $\mathrm{AFM}$ & -68055.4 & 2.045 & 2.060 & 0.77 & -0.77 & 0.05 & 0.40 & 0.5 \\
Configuration I & $\mathrm{FM}$ & -68055.9 & 2.053 & 2.061 & 0.79 & 0.79 & 0.11 & 0.41 & \\
Configuration II & $\mathrm{AFM}$ & -68055.3 & 2.064 & 2.065 & 0.81 & -0.81 & 0.07 & 0.38 & 0.2 \\
Configuration II & FM & -68055.5 & 2.064 & 2.065 & 0.79 & 0.80 & 0.07 & 0.37 & 0.2 \\
\hline
\end{tabular}

As shown in Table 2, we compared $\mathrm{Zn}_{30} \mathrm{Ni}_{2} \mathrm{O}_{32}$ with pure $\mathrm{ZB} \mathrm{ZnO}$, the total energy of the two configurations increased after being doped with $\mathrm{Ni}$ atoms, indicating that the stability of the crystal structure decreased. For configuration I, the energy of the FM state was $0.5 \mathrm{eV}$ lower than the AFM state. While for configuration II, the energy of the FM state was $0.2 \mathrm{eV}$ lower than the energy of the AFM state. The above calculation results show that the ground state of the two configurations of Ni-doped $\mathrm{ZB} \mathrm{ZnO}$ corresponds to a FM state. In particular, it is obvious that the energy differences $(\Delta E)$ increase with the decrease in the length of the $\mathrm{Ni}-\mathrm{O}$ bonds. The largest energy difference $(\Delta E)$ is $0.5 \mathrm{eV}$ corresponding to configuration $\mathrm{I}$. The above results imply that a room temperature ferromagnetism of $\mathrm{Ni}$-doped $\mathrm{ZnO}$ can be expected. In addition, the $\mathrm{Ni}-\mathrm{O}$ and $\mathrm{Zn}-\mathrm{O}$ bond lengths of $\mathrm{Ni}$-doped $\mathrm{ZB} \mathrm{ZnO}$ increased significantly more than the $\mathrm{Zn}-\mathrm{O}$ bond lengths of pure $\mathrm{ZB} \mathrm{ZnO}$, which is primarily due to the geometry optimization lattice distortion caused by $\mathrm{Ni}$ atoms doping. The chemical interaction between $\mathrm{Ni}$ and $\mathrm{ZnO}$ was studied using calculated Mulliken bond populations (Table 2), because bond population can be used to analyze the ionic or covalent bonds [31]. The results indicated that the covalent bond of the doping system became stronger and the ionic bond became weaker than those of pure $\mathrm{ZB} \mathrm{ZnO}$ with the increasing value of Mulliken bond populations; the covalent bond of FM state of configuration I was strongest. We also see in Table 2 that the magnetic moments of $\mathrm{Ni}$ atoms were $0.77 \mu_{\mathrm{B}}$ and $-0.77 \mu_{\mathrm{B}}$ for AFM coupling in configuration $\mathrm{I}$, while the magnetic moment of Ni atoms was $0.79 \mu_{\mathrm{B}}$ for FM coupling, but the calculated magnetic moments were less than the theoretical magnetic moment of $2.0 \mu_{\mathrm{B}}$. The results show that $\mathrm{Ni} 3 d$ states lost electrons at a greater rate; $\mathrm{O}$ atoms around two $\mathrm{Ni}$ atoms also induced a weak magnetic moment, the magnetic moments were $0.05 \mu_{\mathrm{B}}$ and $0.11 \mu_{\mathrm{B}}$. For configuration II, the magnetic moments of the FM and AFM state increased slightly, 
and the $\mathrm{Ni}$ atom and neighbor $\mathrm{O}$ atom had weak magnetic moments. The above results indicate that $\mathrm{Ni}$-doped ZB ZnO has a certain magnetism, which is mainly because the $\mathrm{Ni} 3 d$ state in outer valence electron configuration is $3 d^{8}$; the $\mathrm{Ni}^{2+}$ atom does not introduce extra valence electrons, but $\mathrm{Ni} 3 d$ orbits form a $\mathrm{Ni}^{2+}-\mathrm{O}^{2-}-\mathrm{Ni}^{2+}$ spin polarization with neighboring $\mathrm{O}$ atoms, thus leading to the outer valence electron redistribution.

For comparing and analyzing the influence of the electronic structure and magnetic properties of Ni-doped ZB ZnO, we calculated the band structure and density of states (DOS) of pure ZnO. As can be seen from Figure $2 \mathrm{a}, \mathrm{b}$, the bottom of the conduction band and top of the valence band of pure $\mathrm{ZnO}$ are located at $\Gamma$ point of the Brillouin zone, the calculated band gap is $1.0 \mathrm{eV}$, and showed $\mathrm{ZB} \mathrm{ZnO}$ is a direct wide band-gap semiconductor material, consistent with the existing theoretical results [23,32]. The total DOS of pure ZnO shows that the spin DOS is completely symmetrical, which indicates that pure $\mathrm{ZnO}$ is not magnetic.
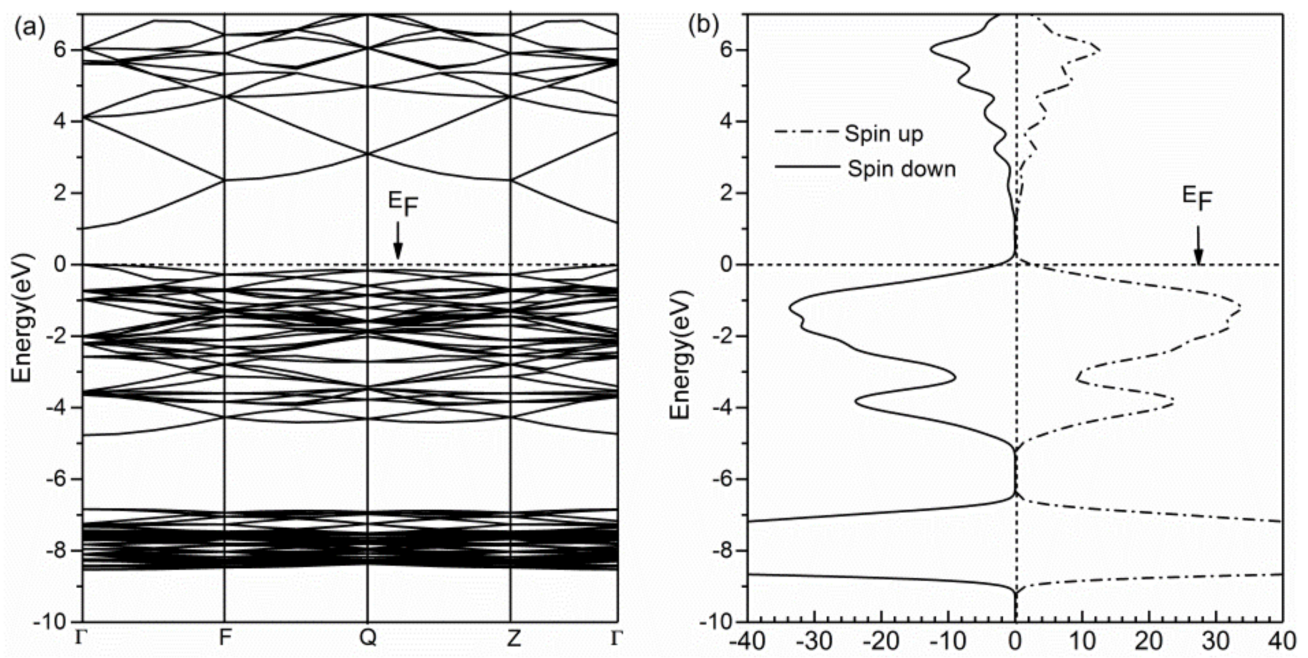

Figure 2. (a) Band structure of pure $\mathrm{ZB} \mathrm{ZnO;} \mathrm{(b)} \mathrm{Total} \mathrm{DOS} \mathrm{of} \mathrm{pure} \mathrm{ZB} \mathrm{ZnO.}$

In order to study the mechanism of magnetic coupling, the calculated band structure of Ni-doped $\mathrm{ZnO}$ will be discussed in Figure 3; this is the band structure of the FM and AFM state for Ni-doped $\mathrm{ZB} \mathrm{ZnO}$. As can be seen from the Figure 3b, the energy levels of spin-up and spin-down are almost the same, the magnetic moment of the AFM state is zero, and the doped system is not magnetic. Moreover, the spin energy levels pass through the Fermi surface, the results exhibit metallic characteristics for the AFM state. In addition, at the top of the valence band, there appeared two and a half filled impurity levels. These impurity levels, which are important for carrier mobility, which is conducive to Ni-doped $\mathrm{ZnO}$ carrier transport, can significantly improve the Ni-doped $\mathrm{ZnO}$ conductive properties. For the band structure of the FM state, it can be seen from Figure 3a, the energy levels of spin-up and spin-down are obviously different in the band structures, the energy level of spin-down electrons has undergone an obvious spin splitting at the Fermi level, and exhibits a strong spin polarization phenomenon. The band structures have asymmetric distribution characteristics, the doped systems show magnetic semiconductor properties. Especially in the Fermi surface, Ni atoms and neighboring $\mathrm{O}$ atoms have strong hybrid interactions, and crystal field effects lead to strong hybrid orbitals splitting, the spin-down energy levels pass through the Fermi surface, and the material appears to be in a semi-metallic FM state. The results indicate that Ni-doped ZB ZnO is a good half-metallic magnetic material. 

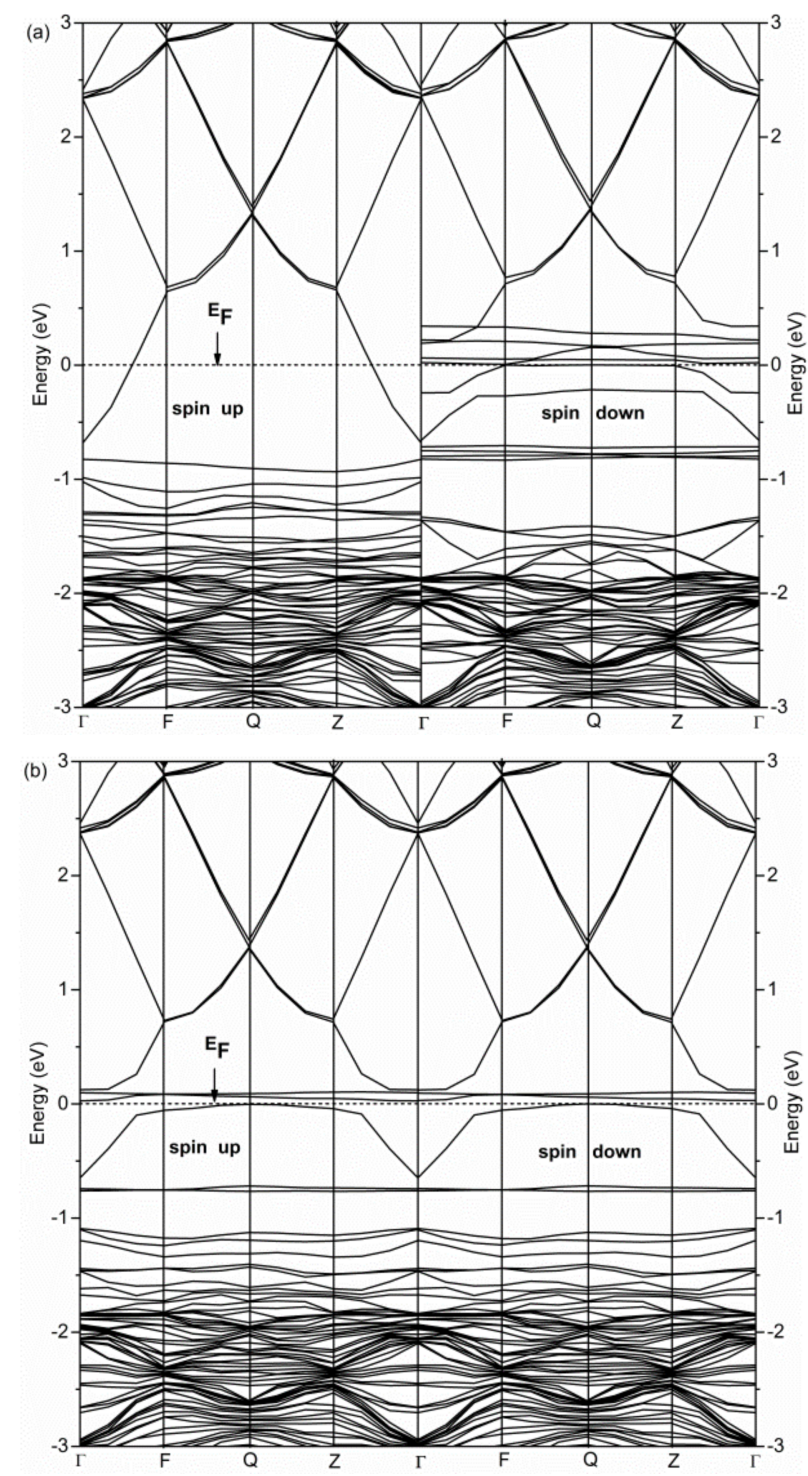

Figure 3. Band structures of Ni-doped ZB ZnO. (a) FM; (b) AFM.

To further explain the magnetic properties of Ni-doped $\mathrm{ZB} \mathrm{ZnO,} \mathrm{we} \mathrm{calculated} \mathrm{the} \mathrm{total} \mathrm{and}$ partial DOS of the FM and AFM states, as shown in Figure 4. As can be seen from Figure 4a, the total and partial DOS are obviously spin-orbit splitting near the Fermi level; the spin-down DOSs of the Ni $3 d$ state at the Fermi level split into two peaks, the spin-up and spin-down DOS underwent a significant shift, there is a clear spin splitting phenomenon. Near the Fermi surface, the spin-up DOS had semiconductor properties, while the spin-down electrons had a metallic property, and it is obvious that the FM system is caused by the $100 \%$ electron-spin polarization and the half-metallic magnetic material. This is mainly due to the $\mathrm{Ni} 3 d^{8}$ orbital electrons between the atoms, forming $\mathrm{Ni}^{2+}-\mathrm{O}^{2-}-\mathrm{Ni}^{2+}$ orbital coupling, resulting in the outermost valence electrons atomic migration. Especially in the neighboring layer, the partially occupied spin-down $\mathrm{Ni} 3 d$ orbital electrons are likely to stabilize the FM state by filling the lower bonding state, and electrons in the Ni $3 d$ state are arranged to form a FM coupling in the same direction. As seen in Figure 4e,f, the DOS of the AFM state is symmetrical, the upper valence band $(-3 \mathrm{eV}-0.0 \mathrm{eV})$ is mainly composed of $\mathrm{Ni} 3 d$ and $\mathrm{O} 2 p$ states with a strong hybridization of orbitals, causing the $\mathrm{Ni} 3 d$ and $\mathrm{O} 2 p$ states to move in a higher energy direction and through the Fermi surface. Therefore, the doped system of the AFM state mainly forms metallic bonds by $\mathrm{Ni} 3 d$ and $\mathrm{O} 2 p$ orbital hybridization. 


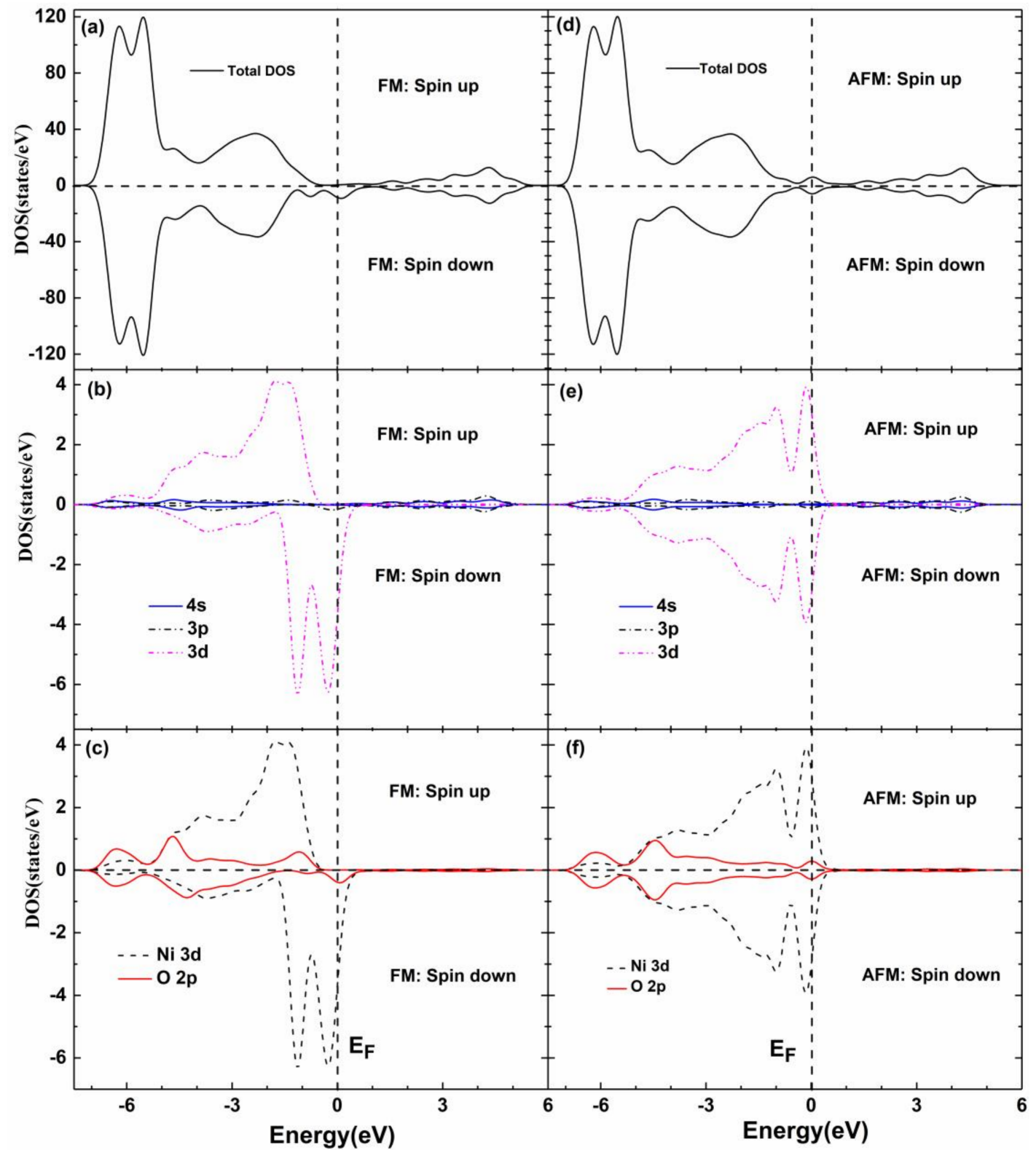

Figure 4. Total and partial DOS of Ni-doped ZB ZnO. (a) total DOS of FM; (b) partial DOS of Ni atoms for FM; (c) partial DOS of Ni $3 d$ and O $2 p$ for FM; (d) total DOS of AFM; (e) partial DOS of Ni atoms for AFM; (f) partial DOS of Ni $3 d$ and $\mathrm{O} 2 p$ for AFM.

To further investigate the coupling mechanism of Ni-doped $\mathrm{ZB} \mathrm{ZnO,} \mathrm{we} \mathrm{approximated} \mathrm{the}$ $\mathrm{Ni}$ atom in a tetrahedral crystal field $\left(T_{d}\right)$, the specific structure is shown in Figure 5. Where the five-fold degeneracy of the $\mathrm{Ni} 3 d$ band was transformed to a three-fold degenerate $t_{2 g}$ state and two-fold degenerate $e_{g}$ states. In accordance with the Hund rule and double exchange mechanism, the calculated results show that a coupling model appeared in the FM and AFM states, when we replaced $\mathrm{Zn}^{2+}$ ions with $\mathrm{Ni}^{2+}$ ions and formed partially occupied $t_{2 g}$ orbital electron. This is the lowest energy state corresponding to the FM state, since four electrons occupy $t_{2 g}$ orbits, four electrons occupy $e_{g}$ orbits. As a result spin electrons of the $\mathrm{Ni}^{2+} e_{g}$ state and neighboring $\mathrm{Ni}^{2+} e_{g}$ states are in the same direction, and $\mathrm{Ni}^{2+}$ ions can be coupled to each other and moved between the two neighboring $\mathrm{Ni}^{2+}$ ions, resulting in Ni-doped ZB ZnO appearing in a stable FM state. The conclusion is consistent with our above-calculated results. 


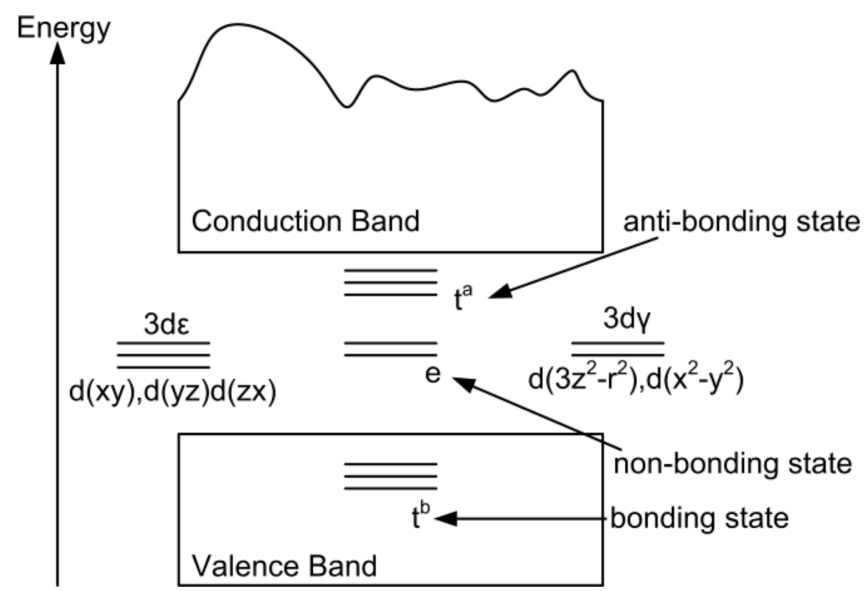

Figure 5. Energy graph of $3 d$ transition-metal atoms in a tetrahedral crystal field $\left(T_{d}\right)$.

\section{Conclusions}

In this study, the electronic structure and coupling mechanism of Ni-doped ZB ZnO are investigated by using the spin polarization approach based on first-principles DFT. By analyzing the energy, magnetic, and electrical properties of different magnetic coupling models, we found that $\mathrm{Ni}$-doped ZB ZnO has a FM and an AFM state. The energy calculated results show that the FM state is more energetically favorable than its AFM state. The results show that $\mathrm{Ni} 3 d$ and $\mathrm{O} 2 p$ have a strong $p-d$ hybridization effect at the Fermi level for the FM coupling state, with $100 \%$ electron-spin polarization and semi-metallic magnetic properties. For the AFM coupling state, the spin-up and spin-down orbits are symmetrically distributed near the Fermi level, and both pass through the Fermi level, with metallic magnetic properties. Moreover, we also found that in the $\mathrm{Ni} 3 d$ orbital in the doping system, there exists a large exchange of splitting and crystal field splitting. The magnetic moment is mainly derived from the construction of the Ni $3 d$ state, with a few parts originating from the neighboring $\mathrm{O} 2 p$ state. The calculated total magnetic moment is related to the doping position of the Ni atoms. Therefore, the calculated results are useful for theoretical instruction and designing stable FM coupling and high Curie temperature of DMS.

Author Contributions: S.X. and F.Z. conceived and wrote this manuscript; S.Z., X.W. and T.S. performed the theoretical calculations and edited the manuscript; F.Z., S.Z. and T.S. analyzed the calculated data; all authors discussed the results and commented on the manuscript.

Acknowledgments: This research was financially supported by the National Natural Science Foundation of China (No. 61664008), the Special Research Funds for Discipline Construction of High Level University Project (No. 2015SXTS02), the Scientific and Technological Innovation Team (2017CXTD-01) and the project of production, learning and research cooperation (2017cxy05).

Conflicts of Interest: The authors declare no conflict of interest.

\section{References}

1. Dietl, T.; Ohno, H.; Matsukura, F.; Cibert, J.; Ferrand, D. Zener Model Description of Ferromagnetism in Zinc-Blende Magnetic Semiconductors. Science 2000, 287, 1019-1022. [CrossRef] [PubMed]

2. Ball, P. Meet the spin doctors. Nature 2000, 404, 918-920. [CrossRef] [PubMed]

3. Wolf, S.; Awshalom, D.D.; Buhiman, R.A.; Daughton, J.M.; von Molnár, S.; Roukes, M.L.; Chtchelkanova, A.Y.; Treger, D.M. Spintronics: A Spin-Based Electronics Vision for the Future. Science 2001, 294, 1488-1495. [CrossRef] [PubMed]

4. Dietl, T. A ten-year perspective on dilute magnetic semiconductors and oxides. Nat. Mater. 2010, 9, 965974. [CrossRef] [PubMed] 
5. Wang, Z.Y.; Liu, Y.Y.; Huang, B.B.; Dai, Y.; Lou, Z.Z.; Wang, G.; Zhang, X.Y.; Qin, X.Y. Progress on extending the light absorption spectra of photocatalysts. Phys. Chem. Chem. Phys. 2014, 16, 2758-2774. [CrossRef] [PubMed]

6. Rekha, K.; BNirmala, M.; Nair, M.; Anukaliani, A. Structural, optical, photocatalytic and antibacterial activity of zinc oxide and manganese doped zinc oxide nanoparticles. Phys. B Condens. Matter 2010, 405, 3180-3185. [CrossRef]

7. Ketsko, V.A.; Beresnev, E.N.; Kop'eva, M.A.; Elesina, L.V.; Baranchikov, A.I.; Stognii, A.I.; Trukhanov, A.V.; Kuznetsov, N.T. Specifics of the pyrohydrolytic and solid-phase syntheses of solid solutions in the $\left(\mathrm{MgGa}_{2} \mathrm{O}_{4}\right)_{x}\left(\mathrm{MgFe}_{2} \mathrm{O}_{4}\right)_{1-x}$ system. Russ. J. Inorg. Chem. 2010, 55, 427-429. [CrossRef]

8. Nipan, G.D.; Ketsko, V.A.; Stognij, A.I.; Trukhanov, A.V.; Kol'tsova, T.N.; Kop'eva, M.A.; Elesina, L.V.; Kuznetsov, N.T. Properties of $\mathrm{Mg}\left(\mathrm{Fe}_{1-x} \mathrm{Ga}_{x}\right)_{2} \mathrm{O}_{4+\delta}$ solid solutions in stable and metastable states. Inorg. Mater. 2010, 46, 429-433. [CrossRef]

9. Kaur, J.; Singhal, S. Facile synthesis of $\mathrm{ZnO}$ and transition metal doped $\mathrm{ZnO}$ nanoparticles for the photocatalytic degradation of Methyl Orange. Ceram. Int. 2014, 40, 7417-7424. [CrossRef]

10. Tian, C.G.; Zhang, Q.; Wu, A.; Jiang, M.; Liang, Z.; Jiang, B.; Fu, H. Cost-effective large-scale synthesis of ZnO photocatalyst with excellent performance for dye photodegradation. Chem. Commun. 2012, 48, 2858-2860. [CrossRef] [PubMed]

11. Sato, K.; Katayamayoshida, H. Material designs for transparent ferromagnetic with $\mathrm{ZnO}$ based magnetic semiconductors. Jpn. J. Appl. Phys. 2014, 39, L555-L558. [CrossRef]

12. Ueda, K.; Tabata, H.; Kawai, T. Magnetic and electric properties of transition-metal-doped ZnO films. Appl. Phys. Lett. 2001, 79, 988-990.

13. Igamberdiev, K.T.; Yuldashev, S.U.; Kwon, Y.H.; Kang, T.W.; Chizhik, S.A.; Trukhanov, A.V.; Rakhimova, S.M. Magnetic phase transitions in $\mathrm{Zn}_{1-x} \mathrm{Mn}_{x}$ O. J. Korean Phys. Soc. 2014, 64, 1457-1460. [CrossRef]

14. Liu, C.; Yun, F.; Morko, H. Ferromagnetism of $\mathrm{ZnO}$ and $\mathrm{GaN}$ : A review. Mater. Sci. Mater. Electron. 2005, 16, 555-597.

15. Zhao, R.B.; Hou, D.L.; Guo, J.M.; Zhen, C.M.; Tang, G.D. Room temperature ferromagnetism in Ni doped ZnO powders. J. Supercond. Nov. Magn. 2010, 23, 1261-1265. [CrossRef]

16. Liu, X.; Lin, F.; Sun, L.; Cheng, W. Doping concentration dependence of room-temperature ferromagnetism for Ni-doped $\mathrm{ZnO}$ thin films prepared by pulsed-laser deposition. Appl. Phys. Lett. 2006, 88, 062508. [CrossRef]

17. Cui, J.B.; Gibson, U.J. Electrodeposition and room temperature ferromagnetic anisotropy of Co and Ni-doped ZnO nanowire arrays. Appl. Phys. Lett. 2005, 87, 133108.

18. He, H., Jr.; Lao, C.S.; Chen, L.J.; Davidovic, D.; Wang, Z.L. Large-scale Ni-doped ZnO nanowire arrays and electrical and optical properties. J. Am. Chem. Soc. 2005, 127, 16376-16377. [CrossRef] [PubMed]

19. Wakano, T.; Fujimura, N.; Morinaga, Y.; Abe, N.; Ahida, A.; Ito, T. Magnetic and magneto-transport properties of ZnO: Ni films. Phys. E 2001, 10, 260-264. [CrossRef]

20. Al-Harbi, T. Hydrothermal synthesis and optical properties of $\mathrm{Ni}$ doped $\mathrm{ZnO}$ hexagonal nanodiscs. J. Alloys Compd. 2011, 509, 387-390. [CrossRef]

21. Cheng, C.; Xu, G.; Zhang, H.; Luo, Y. Hydrothermal synthesis Ni-doped ZnO nanorods with room-temperature ferromagnetism. Mater. Lett. 2008, 62, 1617-1620. [CrossRef]

22. Yin, Z.; Chen, N.; Yang, F.; Song, S.; Chai, C.; Zhong, J.; Qian, H.; Ibrahim, K. Structural, magnetic properties and photoemission study of Ni-doped ZnO. Solid State Commun. 2005, 135, 430-433. [CrossRef]

23. Jaffe, J.E.; Hess, A.C. Hartree-Fock study of phase changes in ZnO at high pressure. Phys. Rev. B 1993, 48, 7903. [CrossRef]

24. Kresse, G.; Furthmiiller, J. Efficiency of ab-initio total energy calculations for metals and semiconductors using a plane-wave basis set. Comput. Mater. Sci. 1996, 6, 15-50. [CrossRef]

25. Perdew, J.P.; Burke, K.; Ernzerhof, M. Generalized gradient approximation made simple. Phys. Rev. Lett. 1996, 77, 3865. [CrossRef] [PubMed]

26. Pfrommer, B.G.; Cote, M.; Louie, S.; Cohen, M.L. Relaxation of crystals with the Quasi-Newton method. J. Comput. Phys. 1997, 131, 233-240. [CrossRef]

27. Jadhav, J.; Biswas, S. Shape-controlled magnetic nanoplatelets of Ni-doped ZnO synthesized via a chemical precursor. J. Alloys Compd. 2016, 664, 71-82. [CrossRef] 
28. Vijayaprasath, G.; Murugan, R.; Mahalingam, T.; Ravi, G. Comparative study of structural and magnetic properties of transition metal $(\mathrm{Co}, \mathrm{Ni})$ doped $\mathrm{ZnO}$ nanoparticles. J. Mater. Sci. Mater. Electron. 2015, 26, 7205-7210. [CrossRef]

29. Grabchikov, S.S.; Trukhanov, A.V.; Trukhanov, S.V.; Kazakevich, I.S.; Solobay, A.A.; Erofeenko, V.T.; Vasilenkov, N.A.; Volkova, O.S.; Shakin, A. Effectiveness of the magnetostatic shielding by the cylindrical shells. J. Magn. Magn. Mater. 2016, 398, 49-53. [CrossRef]

30. Trukhanov, A.V.; Grabchikov, S.S.; Solobai, A.A.; Tishkevich, D.I.; Trukhanov, S.V.; Trukhanova, E.L. AC and DC-shielding properties for the $\mathrm{Ni}_{80} \mathrm{Fe}_{20} / \mathrm{Cu}$ film structures. J. Magn. Magn. Mater. 2017, 443, 142-148. [CrossRef]

31. Mulliken, R.S. Electronic population analysis on LCAO-MO molecular wave functions. J. Chem. Phys. 1955, 23, 1833. [CrossRef]

32. Xu, H.; Sun, W.; Qiu, X.; Wang, L.X.; Yu, M.; Zhang, Q.T. Structural, magnetic and microwave absorption properties of Ni-doped ZnO nanofibers. J. Mater. Sci. Mater. Electron. 2017, 28, 2803-2811. [CrossRef]

(C) 2018 by the authors. Licensee MDPI, Basel, Switzerland. This article is an open access article distributed under the terms and conditions of the Creative Commons Attribution (CC BY) license (http:/ / creativecommons.org/licenses/by/4.0/). 